\title{
Argumentation and Standards of Proof
}

\author{
Katie Atkinson and Trevor Bench-Capon \\ Department of Computer Science \\ University of Liverpool \\ Liverpool L69 3BX UK \\ $\{$ katie,tbc\}@csc.liv.ac.uk
}

\begin{abstract}
In this paper we examine some previous AI and Law attempts to characterise standards of proof, and relate these to the notions of acceptability found in argumentation frameworks, an approach which forms the basis of much recent work on argumentation. We distinguish between the justification of facts and the justification of choices relating to the law and its interpretation. Standards of proof most naturally arise in connection with facts, but points of law have analogous degrees of justification.
\end{abstract}

\section{Keywords}

Argumentation, standards of proof, legal reasoning, purposes.

\section{INTRODUCTION}

It is well known that there are a variety of standards of proof in law: for example, whereas criminal trials require proof beyond a reasonable doubt, civil cases can be settled on the balance of probabilities. These standards of proof have been discussed in AI in Law, for example in [13] and [15]. Recently they have been reconsidered in Gordon and Walton's Carneades Argumentation Framework [16]. In argumentation in AI generally these standards of proof are less used: there we typically find semantic notions of acceptability (such as grounded, preferred and stable, although there are others) originating from Dung [12]. Since these notions, apart from grounded semantics, can give rise to multiple acceptable sets of arguments, we also find the notions of credulous (member of a least one acceptable set) or sceptical (member of all acceptable sets) acceptance.

The above standards relate most naturally to determining the facts of a case. Sometimes, however, decisions require an element of choice, which will depend on the aspirations and interests of the particular person or group of people (audience) making the decision. This aspect of legal decision making, first raised in AI and Law in [9], is discussed in the

Permission to make digital or hard copies of part or all of this work for personal or classroom use is granted without fee provided that copies are not made or distributed for profit or commercial advantage and that copies bear this notice and the full citation on the first page. Copyrights for components of this work owned by others than ACM must be honored. Abstracting with credit is permitted. To copy otherwise, to republish, to post on servers or to redistribute to lists, requires prior specific permission and/or a fee.

ICAIL '07, June 4-8, 2007, Palo Alto, CA USA.

Copyright 2007 ACM 978-1-59593-680-6/07/0006/ ...\$5.00. jurisprudence literature e.g. $[11,18]$ and can reflect the purposes of the law or the prevailing social climate of the society in which the decision takes place. Our work on argumentation draws the distinction between objective (acceptable to all audiences) and subjective (acceptable to some audience) acceptability [5].

In this paper we will consider proposals for characterisations of standards of proof, and relate them to the tools of modern approaches to argument in AI. In section 2 we discuss three accounts that make use of different standards of proof. In section 3 we provide an overview of argumentation frameworks and discuss how we can define different notions of acceptability of arguments based on preferences between arguments, the preferences being justified at a meta-level of argumentation. In section 4 we describe how notions of acceptability can be extended to cover matters of choice as well as matters of fact and why the two are distinct. In section 5 we show how our argumentation-based approach detailed in sections 3 and 4 can be applied to a well-known example from the AI and Law literature concerning the capture of wild animals. In section 6 we demonstrate the approach further through the use of a more recent example case concerning a dispute over possession of a baseball. Finally, we provide some concluding remarks in section 7 .

\section{PROPOSALS FOR STANDARDS OF PROOF}

In this section we will describe three proposals concerning standards of proof from the AI and Law related literature ${ }^{1}$.

\subsection{Freeman and Farley}

In [13] Farley and Freeman present a computational model of dialectical argumentation to serve as a basis for studying legal reasoning. In their account a number of things contribute to the status of an argument. First the authors distinguish a number of warrant types relating premises to conclusions: sufficient, where the premises strictly imply the conclusion; default, where the premises usually imply the conclusion; and evidential, where the premises give some reason to believe the conclusion. Arguments are formed by applying rules with these warrants in one of four ways: modus ponens (MP), which derives the conclusion from the premise; modus tolens (MT), which derives the negation of the premise from the negation of the conclusion; abduction $(\mathrm{ABD})$, where the premise is derived from the conclusion;

\footnotetext{
${ }^{1}$ We do not consider how well these approaches reflect jurisprudential usage.
} 
and abductive contraposition (ABC), where the negation of the premise is derived from the negation of the conclusion. Applying MP or MT to sufficient rules yields a valid argument; applying MP to a default rule yields a strong argument, and applying MP to an evidential rule yields a credible argument. Any other application yields a weak argument. Now a defendable argument is one which cannot be defeated (either by rebuttal or undercut) on the given data. This gives rise to five degrees of support, and the argument may be said to have been shown relative to the standard of proof corresponding to the degree of support. The five degrees of support in [13] are:

- Scintilla of Evidence (se): at least one weak, defendable argument.

- Preponderance of the Evidence (pe): at least one weak, defendable argument that outweighs the other side's argument.

- Dialectical Validity (dv): at least one credible, defendable argument and the other side's arguments are all defeated.

- Beyond a Reasonable Doubt (brd): at least one strong, defendable argument and the other side's arguments all defeated.

- Beyond Doubt (bd): at least one strong, valid argument and the other side's arguments all defeated.

\subsection{The Zeno Framework}

The Zeno system of Gordon and Karacapilidis [15] is used to represent disputes within deliberation dialogues between two individuals whereby the dispute is represented as a dialectical graph. In Zeno there is no notion of strength deriving from warrants and their applications. Rather preferences between arguments are expressed explicitly within the dialectical structure. Zeno structures its dialectical graph around issues, each of which have choices, options for deciding the issues. Each choice has pros and cons, and some constraints expressing preferences between the pros and cons (or the pros of competing choices, or the cons of competing choices). Pros, cons, and constraints may be questioned so that they become sub-issues, each with their own pros, cons, and constraints. Zeno uses five standards of proof:

- Scintilla of Evidence: the choice has some pro.

- Preponderance of Evidence: the pros outweigh the cons given the preference constraints.

- No Better Alternative (nba): no choice is preferred on the basis of the preference constraints.

- Best Choice (bc): one choice is preferred to every alternative choice on the basis of the preference constraints.

- Beyond a Reasonable Doubt: no con reason against a particular choice, and no pro reason for an alternative.

Note that each issue may have a different proof standard. Thus if a con of the main issue must be shown on the preponderance of evidence, then its existence does not rule out the main issue having been shown beyond a reasonable doubt if this standard is not met.
The proof standards of Zeno come in two distinct flavours. Three of the standards, scintilla of evidence, preponderance of evidence and beyond a reasonable doubt, apply to statements of fact, and are similar to three of those from [13]. The remaining two standards, no better alternative and best choice are, however, to do with matters of purpose and preference - things that relate to the aspirations and values of those involved in the debate - and so do not seem happily placed on this scale. Note, however, that in the examples given in [13], facts and interpretation are mixed: all of their warrants seem to relate to the application of intermediate legal concepts, such as responsibility, on the basis of facts which are not in dispute. Thus while the names given to these proof standards seem to suggest that they relate to determining the facts of the case, the examples suggest that they are also meant to apply to the determination of legal concepts, which are normally taken to be questions of law.

\subsection{The Carneades Framework}

Finally we turn to Gordon and Walton's Carneades framework [16], which is a formal, mathematical model of argument structure and evaluation that applies proof standards ${ }^{2}$ to determine the defensibility of arguments and the acceptability of statements. In this framework there are four proof standards which are similar to those proposed in [13], and their names suggest that they do not relate to choices based upon preferences. Such examples as they give seem to relate to questions of fact rather than law. The proof standards of Carneades are:

- Scintilla of Evidence: supported by at least one defensible pro argument.

- Preponderance of Evidence: the strongest defensible pro argument outweighs the strongest defensible con argument, if there is one.

- Dialectical Validity: supported by at least one defensible pro argument and none of the con arguments are defensible.

- Beyond Reasonable Doubt: supported by at least one defensible pro argument, all of its pro arguments are defensible and none of the con arguments are defensible.

The weight of an argument, for the purposes of preponderance of evidence, is given as the weight of its weakest premise.

The question now arises of how these proof standards relate to recent work in argumentation in AI, which has focussed on argumentation frameworks in the style of [12], and their acceptability semantics. In the next section we will focus in particular on the proof standards relating to facts and relate them to Dung-style argumentation frameworks.

\footnotetext{
${ }^{2}$ Discussions with Tom Gordon indicate that the Carneades framework now accommodates a distinction between burden of proof as burden of production and burden of proof as burden of persuasion, as introduced by Prakken and Sartor in [22]. This is an important distinction which we hope to address within our approach in future work [23]. Additionally, "Preponderance of Evidence" is now called "Best Argument".
} 


\section{ARGUMENTATION FRAMEWORKS}

In [12] Dung defined an argumentation framework (AF) as a finite set of arguments $\mathrm{X}$ and a binary relation between pairs of these arguments called an attack. Argument frameworks can conveniently be modelled as directed graphs with arguments as nodes and attacks as edges showing which arguments attack one another. The notion of an argument is entirely abstract: no concern is given to the internal structure of the arguments. Thus, the status of an argument can be evaluated by considering whether or not it can be defended from attack from other arguments with respect to a set of arguments $\mathrm{S} \subseteq \mathrm{X}$. An important notion in Dung's argumentation framework is that of a preferred extension $(\mathrm{PE})$, a subset of the arguments in the framework which:

- is conflict free, in that no argument in the PE attacks any other argument in the PE;

- is admissible, in that the PE is able to defend every argument it contains against attacks from outside the extension: that is, every argument outside the PE which attacks an argument in the PE is attacked by some argument in the $\mathrm{PE}$

- is maximal, in that no other argument can be added to the PE without either introducing a conflict or an argument that cannot be defended against outside attacks.

Another important notion in Dung's AFs is that of the grounded extension (GE), which is similar to the PE except that arguments are not allowed to defend themselves. More formal definitions relating to argumentation frameworks are given in the appendix.

Recall that two of the proof standards of [15] related to matters of subjective preference rather than fact. As discussed in $[4,3]$ such choices can be made by agents according to their purposes, their interests, their aspirations, or the values they wish to promote.

For such arguments we are not concerned with questions of reliability, informedness or honesty, but with the values that will be promoted by acting in accordance with the argument. Since different people may have different value priorities, their choices may differ. Following [19], we refer to groups of people empowered to select arguments on the basis of their values as audiences and characterise audiences in terms of their rankings of the values involved.

On the basis of these notions of values and audiences [5] extended Dung's AFs to enable them to accommodate different audiences with different values and interests. This extension is known as a Value-Based Argumentation Framework (VAF) and it labels arguments with the values that would be promoted if the arguments were accepted. In Dung's AFs an argument is always defeated by an attacker, unless that attacker can itself be defeated ${ }^{3}$. In a VAF, however, attack is distinguished from defeat for an audience whereby strengths of arguments for a particular audience are compared with reference to the values to which they relate. This allows a particular audience to choose to reject an attack, even if the attacking argument cannot itself be defeated. This is on

${ }^{3}$ Besides VAFs there are other approaches to distinguish between attack and defeat using some kind of priorities between arguments, for example, preference-based argumentation frameworks [1]. the proviso that the audience ranks the purpose motivating the attacked argument (the value cited in its justification) as more important than that motivating the attacker. The notion of attack in Dung's PE is then replaced by the notion of defeat for an audience to get the PE for that audience. VAFs are represented as directed graphs, with nodes representing arguments (labelled with an argument identifier, the claim and the value promoted by the conclusion of the argument), and edges representing attacks between arguments.

\subsection{From Dialectical Graphs to Argumenta- tion Frameworks}

Note that in contrast to the work discussed in section 2, neither Argumentation Frameworks nor VAFs have an explicit notion of a pro argument. They recognise only attacking arguments, that is con arguments. Pro arguments can, however, be represented in two ways:

- By attributing structure to the argument: although arguments in Dung are entirely abstract, and in VAFs have only the property of promoting some value, we can attribute structure to them in that to be arguments at all they will take the form reasons, so conclusion. Thus while Zeno, for example, sees an argument as part of a graph where the parent is the conclusion and the child is a supporting reason, both these elements will be inside the node in a argumentation framework or VAF. See [2] for a detailed discussion of how to translate Zeno style dialectical graphs into argumentation frameworks and VAFs.

- By being an attacker of an attacker of the supported argument.

Both these methods were used in [2]. There we showed how the dialectical trees of Zeno can be translated into a VAF representation of the arguments involved. An example of such a translation is given below using the well-known case of Pierson v. Post, which will be described in section 5 . Figure 1 shows the key arguments of the case as represented in a Zeno dialectical tree and Figure 2 shows them transformed into a VAF. Note that in Pierson v. Post the facts were not in dispute, since the court was entirely reliant on the facts as described at the lower court. Support for P1 in Zeno is indicated by the pro of P1, P3, and P7, the con of the preference which leads to the choice for Post being rejected. Both these elements appear in the node of the argument P1/3 in Figure 2; P3 as the relevant circumstances, and P7 as the motivating value. Similarly with the arguments P4 and P6 which support the choice for Pierson. Had P1 been further supported, for example by showing that Post had indeed gained possession of the fox, that could have appeared in Figure 2 as an attacker of $\mathrm{P} 2 / 4$. 


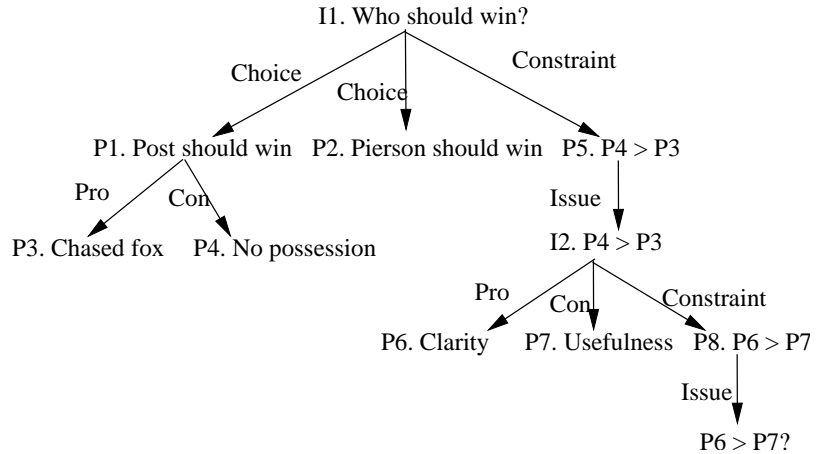

Figure 1: Dialectical Tree from Zeno

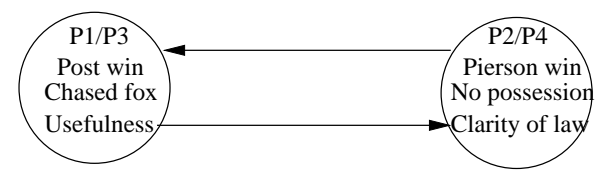

Figure 2: Value-Based Argumentation Framework Version

\subsection{Acceptability and Standards of Proof}

We will resume our discussion of value-based arguments in the next section, but first we return to Dung's AFs to discuss notions of acceptability based on facts. Consider the argumentation framework in Figure 3.

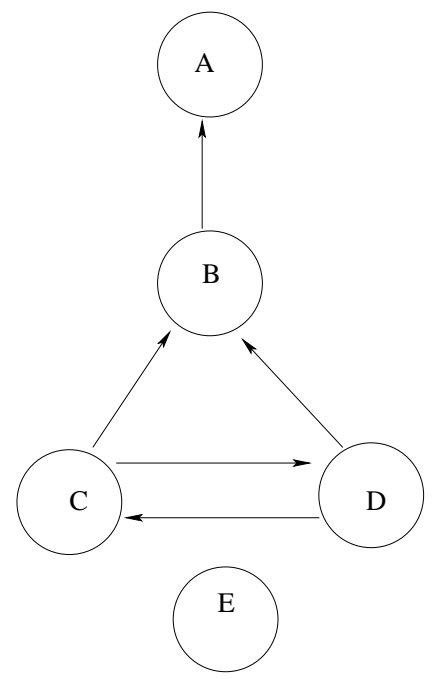

Figure 3. Argumentation Framework

Here we have two preferred extensions, depending on whether we accept $\mathrm{C}$ or $\mathrm{D}:\{\mathrm{A}, \mathrm{C}, \mathrm{E}\}$ and $\{\mathrm{A}, \mathrm{D}, \mathrm{E}\}$. The grounded extension, however, contains only $\mathrm{E}$ since we are unable to defend an argument with itself under grounded semantics, and we are unable to say which of $\mathrm{C}$ or D defends A.

Using the entirely abstract notion of argument found in [12] we can therefore see the following possibilities, remembering that if a grounded extension exists it is unique, and that a grounded extension is also a preferred extension (but not vice versa):

- Some arguments may be in the grounded extension,

- Some arguments may be in all preferred extensions,

- Some arguments may be in some preferred extensions,
- Some arguments may be in no preferred extension.

Note that credulous acceptance implies sceptical acceptance implies membership of the grounded extension. In the argumentation framework in Figure $3 \mathrm{E}$ is in the grounded extension. $\mathrm{A}$ is in all preferred extensions, and hence is sceptically acceptable. E may also be regarded as sceptically acceptable since it too is in all preferred extensions. C and $\mathrm{D}$ are each in one preferred extension and so are credulously acceptable. B is in no preferred extension. Clearly $\mathrm{B}$ is indefensible. $\mathrm{E}$ is entirely secure: there is no reason to doubt it at all. A is relatively secure: it can be defended, although only by an argument of lower status. C and D can be held, in the sense that they cannot be disproved, but can be doubted. We may therefore see the claims of these arguments established in descending order of firmness. We might suggest that they correspond to beyond doubt, beyond reasonable doubt and scintilla of evidence respectively. Note that this leaves a gap where balance of probabilities would be expected: this is because Dung uses no notion of relative strength, so there are no grounds on which we can choose between multiple preferred extensions.

The multiple preferred extensions in an AF arise from the existence of even length cycles [5]. These often arise quite naturally, for example, in cases of incomplete information we may use such a cycle to represent the lack of knowledge as to whether or not some fact holds. At the level of abstraction used by Dung we have no grounds for discriminating between them. We would therefore need to provide a means of discriminating between preferred extensions to be able to supply some standards intermediate between beyond reasonable doubt and scintilla of evidence.

One way of handling this - if the information is available - would be to associate arguments with probabilities. Then when we have to make choices to resolve any even cycles, we could calculate the probability of the resulting preferred extension being the correct choice. In Figure 3, if $\mathrm{C}$ has associated probability 0.7 and $\mathrm{D} 0.3$, the probability of $\{A, C, E\}$ is 0.7 and $\{A, D, E\}$ is 0.3 . Suppose that we add another argument, F, attacking and attacked by E, and both $\mathrm{E}$ and $\mathrm{F}$ have probability 0.5 . Now we have four preferred extensions: $\{\mathrm{A}, \mathrm{C}, \mathrm{E}\},\{\mathrm{A}, \mathrm{C}, \mathrm{F}\},\{\mathrm{A}, \mathrm{D}, \mathrm{E}\}$ and $\{\mathrm{A}, \mathrm{D}, \mathrm{F}\}$ with probabilities, $0.35,0.35,0.15$ and 0.15 respectively. Note that the sceptically accepted A appears in all preferred extensions and the probability sums to 1 . This is because the individual arguments in the two cycles also summed to 1: had $\mathrm{C}$ been 0.7 and D 0.2 , then the probability of A would have been only 0.9 . If the probabilities of arguments in a two cycle do not sum to 1 , this suggests that the options are exclusive, but not exhaustive. In such cases grounded semantics is probably safer, since we cannot supply an argument to represent the remaining alternative, although we acknowledge that there must be one. This kind of approach, based on probabilities for each argument, would certainly be able to give content to the notion of "balance of probabilities". We, however, do not believe that such information is normally available, and will instead talk mainly of a more qualitative approach.

In [2] we suggested that we might label arguments with their source, and that these labels could be used to discriminate cases of conflict. Thus, in Figure 3 suppose that $\mathrm{C}$ was "Witness $\mathrm{W}$ says that $\mathrm{P}$ " and $\mathrm{D}$ was "Witness $\mathrm{V}$ denies that P". Now we will base our decision of whether to accept $\mathrm{C}$ or $\mathrm{D}$ by which of $\mathrm{W}$ or $\mathrm{V}$ we consider to be the 
more credible. Or, in the example of [15] and [2], where the issue is the safety of a particular make of car, one argument may be based on the claims of the manufacturer, and another on the basis of government statistics. We may choose which to believe, although we may be called upon to give a justification for our choice.

Suppose then that our arguments are labelled with their sources, and we give an order for these sources in terms of credibility. As with VAFs, an argument will be defeated by an attacking argument only if its source is not considered more credible than that of the attacker. This will enable us to use the mechanism of VAFs (using the ordering of sources in the same way that VAFs use the ordering on values) described above to determine a unique preferred extension (provided that there are no cycles containing only arguments from a single source). As in [5] we will call an ordering an audience, although the ranking associated with the audience will be one of sources rather than values. Where we need to distinguish we will speak of an audience-for-facts and an audience-for-values. For example suppose that $\mathrm{A}$ and $\mathrm{C}$ are statements of $\mathrm{W}$ and $\mathrm{B}, \mathrm{D}$ and $\mathrm{E}$ statements of $\mathrm{V}$. Now we will accept $\{\mathrm{A}, \mathrm{C}, \mathrm{E}\}$ given $\mathrm{W}>\mathrm{V}$ (read "witness $\mathrm{W}$ is more credible than witness V") and $\{\mathrm{A}, \mathrm{D}, \mathrm{E}\}$ if $\mathrm{V}>\mathrm{W}$ (read "witness $\mathrm{V}$ is more credible than witness W"). Here $\{\mathrm{A}, \mathrm{E}\}$ are accepted independently of the audience (objectively acceptable) while $\mathrm{C}$ and $\mathrm{D}$ depend on the audience (subjectively acceptable). Note that there is no relation between these two notions and sceptical acceptance (this is shown in [6]). Suppose a third witness X, rather than V, testified that B, then the audiences with $\mathrm{X}$ preferred to both $\mathrm{W}$ and $\mathrm{V}$ would accept $\mathrm{B}$ and reject $\mathrm{A}$, reducing all except $\mathrm{E}$ to credulous acceptance.

Of course, the ordering of sources may itself be the subject of argument: in the example above the fact that $\mathrm{V}$ attacks his own argument may lead us to believe W instead. Such recourse is unnecessary for objective acceptance, since the order is immaterial to whether the argument is accepted, but may be useful to determine subjective acceptance if the choice is disputed. A mechanism for producing a hierarchy of argumentation frameworks, in which the orderings determining the acceptable arguments at level $\mathrm{n}$ are debated in an argumentation framework at level $n+1$ is described in [17]. If we have such a meta-level ${ }^{4}$ we can see that orderings may be unjustified, justified for some audiences at the metalevel or justified for all audiences at the meta-level. To avoid confusion we will call these results from the meta-level unsupported, arguable and justified respectively. Thus we may see the choice made at the object level as one of:

\section{- Objectively acceptable}

- Subjectively acceptable for a justified audience

- Subjectively acceptable for an arguable audience

- Subjectively acceptable, but for an audience which is unsupported.

We could perhaps equate these with beyond doubt, beyond reasonable doubt, dialectically valid and scintilla of evidence.

${ }^{4}$ For another treatment of resolving conflicts between arguments at the meta-level see [21].
This treatment, using a single order on sources, has advantages of simplicity, but, unfortunately, it is too simple. On the above approach we are required to be consistent in the ordering throughout the consideration of a case. It does, however, seem perfectly possible to believe one source against another with respect to some disputed facts, and to believe the other source with regard to other facts. It may be, for example, that the two witnesses had different vantage points, so that one could see one aspect clearly and the other a different aspect ${ }^{5}$.

This in turn means that we should not label arguments with their sources at the object level, but instead use the source of an argument as an argument to accept (or reject) it at the meta-level. Here properties of object-level arguments are used as premises in meta-level arguments rather than being used directly at the object level, as in VAFs and preference-based frameworks. Now each conflict as to fact will need to be resolved separately through a meta-level argument, and the outcome of the meta-level will result in each argument being assigned the status justified, arguable or unsupported. We must next remove arguments which have insufficient support. Which these are will depend on the proof standard applicable in the context: for beyond reasonable doubt only justified arguments will be retained, whereas for scintilla of evidence all arguments can be retained. After removing the arguments which have not met the standard of proof, we can consider any remaining conflicts. In these cases we must choose one version of the facts. Arguments which have the status arguable included as a result will have more justification than scintilla of evidence, since we can make a case for their acceptance, but will fall short of acceptance beyond reasonable doubt, since a case can also be made for rejecting them. Arguments which are unsupported at the meta-level will have a lesser status, perhaps scintilla of evidence. The result will be a single set of arguments accepted, which will be justified to a degree represented by its weakest member. If all members of the set are justified we may call these the established facts. If some are no better than arguable we may call them the accepted facts and if some are unsupported an interpretation of the facts.

Thus far we have considered only arguments relating to establishing the facts of a case, rather than the interpretation of law. The aspects of legal decision making that relate to the interpretation of the law are discussed in the next section.

\section{SUBJECTIVE PREFERENCES}

In the previous section we spoke of matters of fact, and it is to these that the proof standards of [13] and [16] most directly relate. For choices about the law and its interpretation we have argued, in [2], [3] and elsewhere, that these choices should be seen as a preference on the motivating values, and that these preferences form an ordering on values, an audience-for-values. All of what has been said about the audience-for-facts applies also to audiences-for-values. We will again have objectively and subjectively acceptable arguments, and we can again argue about the appropriate value orderings at a meta-level, so that the audience-for-values

\footnotetext{
${ }^{5}$ A good discussion and progress towards a formal model of reasoning with and about witness testimonies can be found in $[10]$.
} 
may also be justified, arguable or not justified. This will give rise to four possibilities:

- Objectively acceptable - only choice.

- Subjectively acceptable for a justified audience - best choice.

- Subjectively acceptable for an arguable audience - no better choice.

- Subjectively acceptable, but for an audience which is unsupported - possible choice.

In the case of values we take it here that it is appropriate to insist on a single ordering for the case. Whereas it is quite possible to see part of a witness testimony as reliable and part as unreliable, defending different orderings of the same values is arguably a sign of inconsistency ${ }^{6}$.

Typically an argumentation framework will contain both arguments relating to matters of fact and arguments relating to matters of value. Thus an audience for such a framework will in general be a pair <set of facts, order of values $>$.

Depending on the status of the set of facts and the degree of justification of the value order, we may distinguish the following possibilities:

- possible choice on an interpretation of the facts,

- possible choice on the accepted facts,

- possible choice on the established facts,

- no better choice on an interpretation of the facts,

- no better choice on the accepted facts,

- no better choice on the established facts,

- best choice on an interpretation of the facts,

- best choice on the accepted facts,

- best choice on the established facts,

- only choice on an interpretation of the facts,

- only choice on the accepted facts,

- only choice on the established facts.

These range from requiring an interpretation of the facts and an ordering of values which cannot be supported in order to defend an argument, to arguments which are justified whatever the view taken on the facts and values. In the first case we must depend on a hunch and a whim, whereas in the second we have no choice but to accept the argument.

Separating the questions of fact from the questions of law is standard practice in legal decision making. Typically a decision will begin with a statement of the facts, justifying the choice in cases of conflict and will then go on to explain and justify the choices made by the judge as to the matters of law. Also there is often a different mechanism for deciding matters of fact (e.g. juries), and different rules apply to what may be appealed (e.g. an appeal can only be on a matter of law and not on a matter of fact, unless new evidence can be introduced, or it can be established that no reasonable person could have come to the factual conclusion in question).

${ }^{6}$ Here we follow the normal practice in VAFs. Extensions to allow for multiple orderings in a single framework are under current investigation [23].

\section{EXAMPLE}

We illustrate our approach, as discussed in the previous two sections, through use of an example. For this example we will consider the domain of wild animals as discussed widely in the AI and Law literature by e.g. [7, 8, 20], but slightly adapted to make room for some factual arguments. In [7] three cases were considered all of which concern the disputed pursuit of wild animals. The facts of the three cases are:

- Keeble v. Hickeringill (1707). This was an English case in which Keeble owned a duck pond, to which he lured ducks, which he shot and sold for consumption. Hickeringill, whose land adjoined Keeble's, out of malice, scared the ducks away by firing guns. The court found for Keeble.

- Pierson v. Post (1805). In this New York case, Post was hunting a fox with hounds on unoccupied wasteland. Pierson intercepted, killed and carried off the fox. The court found for Pierson.

- Young v. Hitchens (1844). In this English case, both parties were commercial fisherman. Young was out fishing, spread a net of 140 fathoms in open water and began to close it. When the net was almost closed to no more than a few dozen feet wide Hitchens sped into the remaining gap. Hitchens then spread his own net and caught the fish which had been trapped by Young as he closed his net. The case was decided for Hitchens.

In our discussion we will focus our attention on the final case in this list, Young v. Hitchens, using arguments presented in the AI and Law literature (mainly [9]). We will now represent the arguments involved in this case in terms of a VAF. Firstly, the plaintiff needs to prove his case, and so we begin the argument that we should find for the defendant. Young must now find arguments which defeat that claim, and Hitchens must defeat those arguments. We now have to consider the arguments which might be presented.

Young argues:

Y1: I had been drawing in my nets for three hours. This is a factual claim.

Y2: The fish had no prospect of escape. Factual claim, needed as premise for Y3.

Y3: They were my fish because they had no prospect of escape. Granting possession with this degree of control encourages fishing.

Y4: Hitchens should be punished as he is guilty of taking my fish. Claimed consequence.

Y5: Hitchens should be punished as unfair practices such as his threaten the fishing industry. Appeal to the value of encouraging fishing.

Hitchens counters:

H1: Young's nets were breaking: the fish were about to escape. Factual claim, denying Y2, needed as premise of Y3.

$\mathrm{H}$ 2: The fish were available for capture since they had not been caught. Bright line argument based on need for clarity. H3: Competition must be expected in business. Setting up the value for $\mathrm{H} 4$.

H4: I should not be punished as that would inhibit vigorous competition. Counter appeal to value of competition. 
This gives rise to the VAF shown in Figure 4.

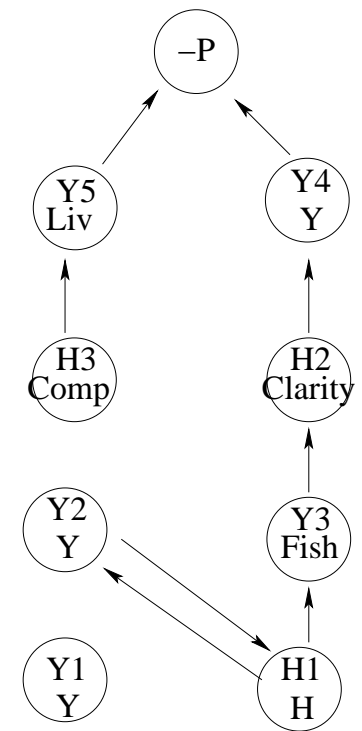

Figure 4. VAF for Young v. Hitchens

The fact $\mathrm{Y}^{7}$ is not disputed and so must be accepted. The fact H1, if accepted will defeat $\mathrm{Y} 3$, leaving $\mathrm{H} 2$ to attack Y4. So first the factual dispute over the state of the nets must be resolved. At the meta-level, we might ideally want to make physical investigation of the actual nets. If the nets are unavailable we simply have to decide who is more credible. We could argue that we should, in the absence of concrete physical evidence to the contrary, presume that Young, a professional fisherman, will have maintained his nets, and so require more than Hitchens' unsupported statement. Moreover we can argue that Hitchens may well be biased, giving the set of arguments shown in Figure 5 below. On the basis of these arguments, we will accept Y2, although this is not fully justified, but merely arguable: we could simply assert that Hitchens is to be believed despite his bias ${ }^{8}$. Accepting Y2 will be enough to establish it for the civil proceedings at hand, but it might not suffice for criminal proceedings where Y2 would need to be established beyond reasonable doubt. Still we can take Y1 and Y2 as the accepted facts, whereas someone who insists on believing Hitchens could only claim $\mathrm{H} 1$ and $\mathrm{H} 2$ as an interpretation of the facts.

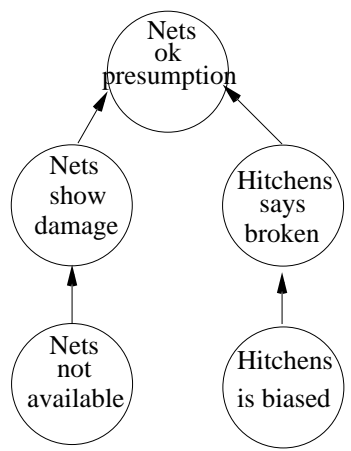

Figure 5. Meta-level arguments about facts

${ }^{7} \mathrm{Y} 1$ and $\mathrm{H} 1$ are additions to the arguments on our part, provided to give examples of contested facts.

${ }^{8}$ This relates to the distinction between burden of production and burden of persuasion [22].
We next need to make a value comparison: should we prefer the flexibility to encourage fishing or clarity? Again we must ascend to the meta-level, this time to resolve the ranking of values. Now we will need not more factual evidence, but some indication of which is the right ordering of the competing values, and here precedent becomes relevant. At this level it is possible to cite precedents to show that a particular ordering was used in the past, authorities to give weight to adopting a particular order, or to appeal to a teleological argument to justify the order. Thus, for authorities Hitchens can cite Justinian, and Young can cite Barbeyrac, both discussed in the Pierson case. Hitchens can cite Pierson itself, while Young can give Keeble as a counter example. Hitchens may distinguish Keeble, on the grounds that Keeble was on his own land, and this was the reason to grant him possession of the ducks. Young may also make a teleological argument that finding for him will be good for the fishing industry, which may be countered by the argument from [9] that deeming Young to have possession in the absence of explicit legislation or the kind of accepted trade conventions that exist in whaling would lead to endless disputes.

The argumentation at the meta-level may look as in Figure 6 .

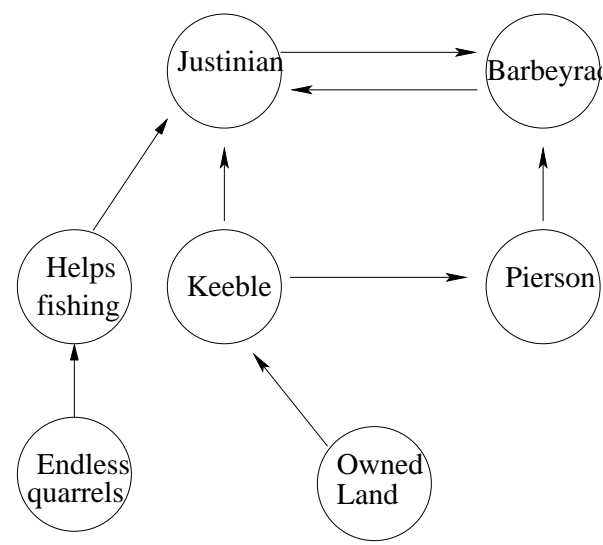

Figure 6. Meta-level arguments about value order

The essential dispute here is whether the distinguishing move works, and this depends on how one interprets the decision in Keeble. If one felt that it was the economic benefits of allowing Keeble to ply his trade that were decisive (the interpretation that seems to be favoured by [9] and which can be supported by some passages in the decision), then Keeble will support Young. It is still, however, possible to resist this argument: Justinian is an ancient authority and it may be argued that old authorities are best (alternatively it could be argued that Justinian was so old as to be no longer relevant, a point made in the minority opinion of Pierson). Other passages, however, and the use made by Tomkins of Keeble in the majority decision in Pierson, suggest that the distinction is effective. This leaves us with no evidence that Barbeyrac can overrule Justinian. We may now consider Young's teleological argument, which turns on whether the unhindered pursuit of livelihood is worth the endless quarrels that might result. It appears that the judges thought not. Thus the preference of clarity over encouraging fishing is justified at the meta-level, and so $\mathrm{H} 2$ is the best choice on the accepted facts. If we accept $\mathrm{H} 2$, then we must abandon Y4. Note that this is not because of a preference, but 
because $\mathrm{Y} 4$ requires the conclusion of $\mathrm{H} 2$ to be false if its premises are to hold: accepting $\mathrm{H} 2$ thus destroys $\mathrm{Y} 4$ as an argument and it can play no further role.

The final try by Young is to make his teleological argument, Y5, at the object level and argue that fisherman should be allowed to go about their business without fear of interference such as was offered by Hitchens, even if it cannot be considered that they are in possession of the fish. Hitchens counters that vigorous competition should be encouraged. Here again the judges are being asked to express a preference, and the endless quarrels argument accepted previously will again serve to justify rejecting Young's argument. Thus although Young has a case that can be made for some audiences, Hitchens is able to justify the orderings favouring him, and so the judges dismissed the claim.

\section{POPOV V. HAYASHI}

In order to see the current relevance of these rather old cases, we will consider the 2002 case Popov v. Hayashi, decided by the Honourable Kevin M McCarthy in the Superior Court of California (no. 400545), and also discussed by Finkelman in [14].

The case concerned the possession of the baseball which was struck to give Barry Bonds his record breaking 73rd home run in the 2001 season. Such a ball is very valuable (Mark McGwire's 1998 70th home run ball sold at auction for $\$ 3,000,000)$. When the ball was struck into the crowd, Popov caught it in the upper part of the webbing of his baseball glove (fans often wear baseball gloves to assist in catching balls that leave the park). Such a catch - known as a snowcone catch - where the ball is not fully in the mitt, does not give certainty of retaining control of the ball, particularly since Popov was stretching and may have fallen. Popov was not, however, given the chance to complete his catch since, as it entered his glove, he was tackled and thrown to the ground by others trying to secure the ball. In the ensuing scrum it was dislodged from the glove and picked up by Hayashi (himself innocent of the attack on Popov), who put it in his pocket, so securing possession.

The relation with wild animals is obvious and Pierson and Young were among the precedents cited. As Finkelman says:

... the home run ball, once it goes over the fence, is like a wild, unclaimed creature. It is much like a fox, elk, or whale, being hunted, not by people with guns or harpoons, but by fans with gloves.

The question, as in Young, was whether Popov had possession of the ball. McCarthy is clear that if Popov would have retained control of the ball but for the interference of the crowd, then he would have a right to possession. However, says McCarthy:

We will never know if Mr. Popov would have been able to retain control of the ball had the crowd not interfered with his efforts to do so. Resolution of that question is the work of a psychic, not a judge.

However, unlike Young, the act which prevented Popov from attempting to perfect his control was illegal: he was assaulted by a mob. This, for McCarthy, gave Popov a "legally cognizable pre-possessory interest" in the ball: he should have been given a fair opportunity to complete his catch. This did not, however, defeat Hayashi's claim, based on the rights of a finder, since the established pre-possessory interest of Popov did not establish a full right to possession protected from a subsequent legitimate claim. McCarthy's final verdict was a judgement of Solomon: although he did not actually ask for the ball to be cut in half, he decided that it should be sold and the proceeds divided between the two sides.

Finkelman in [14] comes to a different answer. He thinks that Popov did possess the ball, but he does so on a different interpretation of the facts. He claims:

Films clearly show Popov with the ball in his glove, after which he was covered by a crowd of fans.

Finkelman dismisses the opinion of baseball umpire Rich Garcia, who argues that a snowcone catch is not a fair catch, since the ball is not yet fully under control, and if it is then dropped it is no catch. Had McCarthy agreed with him as to the facts, he would have agreed with him on the judgement, but as we have seen he did not believe the catch to be complete before the mob hit Popov. Finkelman also has a teleological argument:

... if the ball bounces out because it is forced out by others, or pulled out by others, then the default rule must be that the person who first had it in his glove is the owner. Otherwise, the rule of law is replaced with the rule of the jungle. If people who do not catch the ball can claim it by forcing it out of the glove of the fan who did catch it - as is the case with Popov - then the "rule" allowing this will encourage violence and mayhem. No fan can be safe with a ball because the next fan, the bigger fan, can jostle it free.

Finkelman believes that Popov had full control of the ball, and, if this were so, the case would have been clear: the wild animal precedents suffice to justify attributing possession to Popov. But McCarthy's assessment of the facts was different: Popov's catch was interrupted by the illegal assault before it was completed. Finkelman could still advance his teleological argument to suggest finding for Popov, but this would no longer be supported by the wild animal precedents since possession is not held to be complete. McCarthy could choose to accept this, and he does accept the value as worth promoting, but in his view, the value can be promoted by means other than finding wholly for Popov: he does this by his recognition of the pre-possessory right.

McCarthy's decision is an excellent illustration of the process we have described in the preceding sections. It is explicitly structured into two distinct parts: facts and legal analysis. In the facts part he evaluates the conflicting testimony of seventeen witnesses, together with videotape footage. He indicates some of his criteria: differences in vantage point, interest in the outcome, quality of memory, consistency. Generally McCarthy's most preferred source is the videotape. He indicates this ordering by saying at one point:

The videotape clearly establishes that this was an out of control mob, engaged in violent, illegal behaviour. Although some witnesses testified in a manner inconsistent with this finding, their testimony is specifically rejected as being false on a material point. 
At another point concerning Popov's contention that Hayashi bit a Mr. Shepard, McCarthy states:

The tape does not support such a conclusion. The testimony which suggests a bite occurred is equally unconvincing.

The conflicts in evidence are identified, and a version chosen for each conflict, usually with a justificatory argument. In the legal analysis McCarthy similarly proceeds by identifying a series of issues which he resolves with a choice justified either by authority (sometimes written, sometimes taken from a specially convened forum of four Law Professors) or by citation (Pierson and Young are included among the cites). Finally he endorses Finkelman's teleological argument, albeit to the limited extent of motivating his finding of a pre-possessory right:

\begin{abstract}
His efforts to establish possession were interrupted by the collective assault of a band of wrongdoers. A decision which ignored that fact would endorse the actions of the crowd by not repudiating them. Judicial rulings, particularly in cases which receive media attention, affect the way people conduct themselves. Thus case demands vindication of an important principle. We are a nation governed by law, not brute force.
\end{abstract}

Thus McCarthy accepts the teleological argument, but uses it not to stretch the law regarding possession, but only to the extent of not accepting Hayashi's claim to sole rights to the ball. Does this conflict with the view that a consistent order on values is required, since clarity is preferred over public order when dismissing Popov's claim, whereas public order is given precedence when dismissing Hayashi's claim? Arguably not, because Hayashi cannot argue that clarity is served by awarding him the ball: the videotape clearly showed - at least it was accepted by McCarthy that it clearly showed - that Popov was in a position to attempt to complete his catch. It is the relevance of this clearly established fact that matters: there is no unclarity here, at least with respect to the pre-possessory right.

\section{CONCLUDING REMARKS}

In this paper we have related a number of proof standards found in the literature to the acceptability semantics of formal argumentation frameworks. The most often quoted standards relate to matters of fact. These standards can be expressed in terms of: (1) the status of the objectlevel arguments for a fact, and (2) where the acceptability of an object-level argument depends upon a choice, the status of the meta-level arguments justifying membership of an audience licensing this choice. It is noted that some proof standards relate not to facts, but to legal preferences: these can be treated in a similar fashion, although now the audience will represent a ranking of values rather than considerations relating to the reliability of information and testimony.

We have illustrated the process by an example much discussed in the AI and Law literature, and by consideration of a recent related decision, Popov v. Hayashi. This decision in particular proceeds by the identification of conflicts and justifies a series of choices of preferred sources regarding disputed facts, and the subsequent use of authority, precedent and purpose at the meta-level to justify choices as to points of law.

\section{Appendix: Argumentation Framework Defini- tions}

An Argumentation Framework $(A F)$ is a pair $A F=<X$, $A>$, where $X$ is a set of arguments and $A \subset X \times X$ is the attack relationship for $A F$. $A$ comprises a set of ordered pairs of distinct arguments in $X$. A pair $\langle x, y\rangle$ is referred to as " $x$ attacks (or is an attacker of) $y$ " or " $y$ is attacked by $x$ ". For $R, S$, subsets of $X$, we say that:

- $s \in S$ is attacked by $R$ if there is some $r \in R$ such that $<r, s>\in A$.

- $x \in X$ is acceptable with respect to $S$ if for every $y \in$ $X$ that attacks $x$, there is some $z \in S$ that attacks $y$ (i.e. $z$, and hence $S$, defends $x$ against $y$ ).

- $S$ is conflict free if no argument in $S$ is attacked by any other argument in $S$.

- A conflict free set is admissible if every argument in $S$ is acceptable with respect to $S$.

- $S$ is a preferred extension if it is a maximal (with respect to set inclusion) admissible subset of $X$.

- $S$ is a stable extension if $S$ is conflict free and every argument $y, \neg(y \in S)$, is attacked by $S$.

- $S$ is a complete extension if $S$ is a subset of $A, S$ is admissible, and each argument which is defended by $S$ is in $S$.

- $S$ is a grounded extension if it is the least (with respect to set inclusion) complete extension.

- An argument $x$ is credulously accepted if there is some preferred extension containing it.

- An argument $x$ is sceptically accepted if it is a member of every preferred extension.

\section{Acknowledgements}

We acknowledge partial support received from the European Commission through Project ESTRELLA (IST-2004027655). We are also grateful to Tom Gordon for interesting discussions concerning the Carneades framework. Finally, we would like to thank the anonymous reviewers for their helpful comments and suggestions.

\section{REFERENCES}

[1] L. Amgoud and C. Cayrol. Inferring from inconsistency in preference-based argumentation frameworks. Journal of Automated Reasoning, 29(2):125-169, 2002.

[2] K. Atkinson and T. Bench-Capon. Zeno revisited: Representation of persuasive argument. In T. van Engers, editor, Legal Knowledge Information Systems. JURIX 2006: The Nineteenth Annual Conference, volume 152 of Frontiers in Artificial Intelligence and Applications, pages 1-10. IOS Press, 2006.

[3] K. Atkinson, T. Bench-Capon, and P. McBurney. Arguing about cases as practical reasoning. In Proceedings of the Tenth International Conference on Artificial Intelligence and Law (ICAIL 2005), pages 35-44. ACM Press, 2005. 
[4] K. Atkinson, T. Bench-Capon, and P. McBurney. Computational representation of practical argument. Synthese, 152(2):157-206, 2006.

[5] T. Bench-Capon. Persuasion in practical argument using value based argumentation frameworks. Journal of Logic and Computation, 13(3):429-48, 2003.

[6] T. Bench-Capon, S. Doutre, and P.E. Dunne. Audiences in argumentation frameworks. Aritificial Intelligence, 171(1):42-71, 2007.

[7] T. Bench-Capon and E. L. Rissland. Back to the future: dimensions revisited. In B. Verheij, A. Lodder, R. Loui, and A. Muntjewerff, editors, Legal Knowledge Information Systems. JURIX 2001: The Fourteenth Annual Conference, volume 70 of Frontiers in Artificial Intelligence and Applications, pages 41-52. IOS Press, 2001.

[8] T. Bench-Capon and G. Sartor. A model of legal reasoning with cases incorporating theories and values. Artificial Intelligence, 150(1-2):97-143, 2003.

[9] D. H. Berman and C. D. Hafner. Representing teleological structure in case-based legal reasoning: the missing link. In Proceedings of the Fourth International Conference on Artificial Intelligence and Law (ICAIL 1993), pages 50-59. ACM Press, 1993.

[10] F. Bex, H. Prakken, C. Reed, and D. Walton. Towards a formal account of reasoning about evidence: Argumentation schemes and generalisations. Artificial Intelligence and Law, 11(2-3):125-165, 2003.

[11] C. G. Christie. The Notion of an Ideal Audience in Legal Argument. Kluwer Academic Press, 2000.

[12] P. M. Dung. On the acceptability of arguments and its fundamental role in nonmonotonic reasoning, logic programming and n-person games. Artificial Intelligence, 77:321-357, 1995.

[13] A. M. Farley and K. Freeman. Burden of proof in legal argumentation. In Proceedings of the Fifth International Conference on Artificial Intelligence and Law (ICAIL 1995), pages 156-164. ACM Press, 1995.

[14] P. Finkelman. Fugitive baseballs and abandoned property: Who owns the home run ball? Cardozo Law Review, 23(5):1609-1633, 2002.

[15] T. F. Gordon and N. I. Karacapilidis. The Zeno argumentation framework. In Proceedings of the Sixth International Conference on Artificial Intelligence and Law (ICAIL 1997), pages 10-18. ACM Press, 1997.

[16] T. F. Gordon and D. N. Walton. The Carneades argumentation framework: Usuing presumptions and exceptions to model critical questions. In P. E. Dunne and T. Bench-Capon, editors, Computational Models of Natural Argument, Proceedings of COMMA 2006, volume 144 of Frontiers in Artificial Intelligence and Applications, pages 195-207. IOS Press, 2006.

[17] S. Modgil. Value based argumentation in hierarchical argumentation frameworks. In P. E. Dunne and T. Bench-Capon, editors, Computational Models of Natural Argument, Proceedings of COMMA 2006, volume 144 of Frontiers in Artificial Intelligence and Applications, pages 297-308. IOS Press, 2006.

[18] C. Perelman. Justice, Law, and Argument. D. Reidel Publishing Company, Dordrecht, The Netherlands, 1980.

[19] C. Perelman and L. Olbrechts-Tyteca. The New
Rhetoric: A Treatise on Argumentation. University of Notre Dame Press, Notre Dame, IN, USA, 1969.

[20] H. Prakken. An exercise in formalising teleological case-based reasoning. In J. Breuker, R. Leenes, and R. Winkels, editors, Legal Knowledge Information Systems. JURIX 2000: The Thirteenth Annual Conference, volume 64 of Frontiers in Artificial Intelligence and Applications, pages 49-58. IOS Press, 2000.

[21] H. Prakken, C. Reed, and D. Walton. Dialogues about the burden of proof. In Proceedings of the Tenth International Conference on Artificial Intelligence and Law (ICAIL 2005), pages 115-124. ACM Press, 2005.

[22] H. Prakken and G. Sartor. Presumptions and burdens of proof. In T. van Engers, editor, Legal Knowledge Information Systems. JURIX 2006: The Nineteenth Annual Conference, volume 152 of Frontiers in AI and Applications, pages 21-30. IOS Press, 2006.

[23] A. Wyner and T. Bench-Capon. Towards an extensible argumentation system. Technical Report ULCS-07-003, Department of Computer Science, University of Liverpool, Liverpool, UK, 2007. 